\title{
Hybrid MAC protocol for IEEE 802.11 wireless LANs with hidden node problem
}

\author{
Woo-Yong Choi ${ }^{*}$
}

\begin{abstract}
Combining the IEEE 802.11 basic MAC (medium access control) protocols, which are the DCF (distributed coordination function) and the PCF (point coordination function), we propose a hybrid MAC protocol to improve the performance of IEEE 802.11 wireless LANs and mitigate the hidden node problem.

K e y w o r d s: wireless LAN, MAC, throughput, DCF, PCF
\end{abstract}

\section{Introduction}

The DCF (distributed coordination function) suffers from the exponential performance degradation as the number of active nodes grows, and the performance of the other basic MAC (medium access control) protocol, which is the PCF (point coordination function), worsens as the smaller portion of nodes are active because unnecessary polling and response frames are transmitted between inactive nodes and AP (access point) [1,2]. The hidden node problem that exists in IEEE 802.11 wireless LANs further aggravates the performance of the DCF [3]. Exploiting the contrasting characteristics of the DCF and the $\mathrm{PCF}$ in MAC performance, the hybrid approach combining the DCF and the PCF is promising to resolve the problem of IEEE 802.11 wireless LANs and improve the MAC performance.

One approach for mitigating the hidden node problem of IEEE 802.11 wireless LANs is to divide nodes into multiple clusters so that no hidden node problem exists in each cluster and assign distinct time slots or orthogonal frequency slots to clusters $[4,5]$. However, the exponential performance degradation of the DCF due to the huge number of active nodes in IoT (internet of things) traffic environment was not considered in $[4,5]$. In [6], combining the DCF and the PCF, the clustering method for resolving the performance degradation problem due to the huge number of active nodes was proposed without the consideration of the hidden node problem. In this paper, combining the DCF and the PCF, for resolving the hidden node problem and the exponential performance degradation simultaneously, we propose an unifying hybrid MAC protocol with the new clustering method.

\section{Unifying hybrid MAC protocol}

According to [2], MAC service period is divided into CFPs (contention free periods) where the PCF is operated and CPs (contention periods) where the DCF is operated, and CFPs and CPs are alternated with each other. AP can estimate the uplink MAC throughput as the total bits of payloads in MAC frames successfully transmitted from nodes over total MAC operation time.

During CFPs, AP collects the PHY carrier sensing information among nodes. The carrier sensing information $C_{i, j}$ between nodes $i$ and $j$ is

$$
C_{i, j}= \begin{cases}1 & \begin{array}{l}
\text { if node } j \text { senses the PHY signal } \\
\text { transmitted from node } i
\end{array} \\
0 \quad \text { otherwise }\end{cases}
$$

Nodes update $C_{i, j}$ 's by continuously hearing the signals from other nodes during CFPs. For collecting the carrier sensing information from nodes, AP sends polling frames to nodes, and receives from nodes response frames containing the changes of the carrier sensing information. This method for collecting the carrier sensing information was employed to mitigate the hidden node problem in [4].

Now, we want to explain the new clustering method, which can be implemented in AP using the carrier sensing information, for resolving the hidden node problem and the DCF performance degradation simultaneously. Let us assume that AP tries to cluster $n$ nodes into $m$ clusters. The clustering method begins with m empty bins where each bin represents a cluster. Selecting a random node that has not yet been inserted into a bin, AP attempts to insert the selected node into a bin. Let us assume that

* Department of Industrial and Management Systems Engineering, Dong-A University Nakdong-daero, Saha-gu, Busan, Korea, wychoi77@dau.ac.kr 


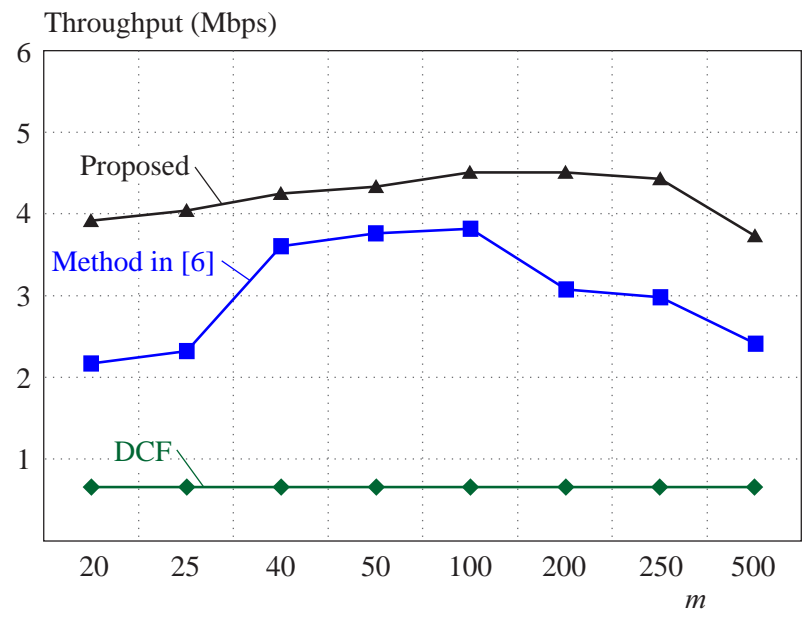

Fig. 1. MAC throughput results

Table 1. Values of simulation parameters

\begin{tabular}{lc}
\hline Parameters & Values \\
\hline Data Trans. Rate & $6.5 \mathrm{Mbps}$ \\
ACK Trans. Rate & $6 \mathrm{Mbps}$ \\
SIFS & $16 \mu \mathrm{s}$ \\
DIFS & $34 \mu \mathrm{s}$ \\
Time Slot & $9 \mu \mathrm{s}$ \\
Max. Backoff Stage & 7 \\
PHY Header Trans. Length & $20 \mu \mathrm{s}$ \\
CP Length, CFP Length & $100 \mathrm{~ms}$ \\
\hline
\end{tabular}

AP attempts to insert node $k$ into bin 1 . The insertion can be successful if

$$
\begin{aligned}
& C_{p, k}=C_{k, p}=1 \\
& \text { for each node } p \text { existing in bin } l
\end{aligned}
$$

so that no hidden node problem exists among nodes in bin $l$. If the attempt of the insertion of node $k$ into bin $l$ fails, AP continuously attempts to insert node $k$ into the next bins in the order of increasing size of bin, which is the number of nodes existing in bin. By the order of increasing size of bin by which AP attempts the insertions, we can have the resulted clusters with the sizes that are as even as possible. When during the clustering process AP cannot find bin $l$ among $m$ bins satisfying the condition (2) for any node $k$ that has not yet been inserted into a bin, incrementing the number of clusters, AP should retry to cluster $n$ nodes into $m+1, m+2, \ldots, n$ clusters sequentially until $n$ nodes are clustered successfully.

Polling nodes and receiving responses from nodes during the previous CFPs where each node is granted at least one transmission opportunity using the PCF, AP can determine whether each node is active or not. To distribute active nodes over the clusters evenly, AP should try to in- sert active nodes into bins first and insert inactive nodes afterwards.

If AP succeeds in clustering nodes into $m$ clusters, AP can divide CP into $m$ SPs (sub-periods) of equal length, each of which is assigned to a cluster. By the proposed clustering method, no hidden node problem exists within each cluster, and the exponential performance degradation can be resolved because of the reduced number of nodes that participate in the DCF contention. As discussed in [4], according to channel state, during CFPs AP can piggyback the cluster numbers on polling frames to inform nodes of the updated clusters to which nodes belong. At the start of each SP during CPs, AP should broadcast announcement frame with the corresponding cluster number an SIFS (short inter-frame space) period after the previous frame exchange sequence, and only the nodes belonging to the cluster contend for their transmissions using the DCF during the assigned SP. However, if only a single node composes a cluster in a extreme case, AP grants the transmission opportunity using the PCF during the assigned SP.

Continuously monitoring the MAC performance for various values of $m$ and changing $m$ according to channel state in the direction of increasing the MAC performance, we can obtain the appropriate hybrid MAC protocol. As the portion of active nodes becomes larger, the appropriate hybrid MAC protocol becomes resembling the PCF with larger $m$. As the portion of active nodes smaller, the hybrid MAC protocol resembling the DCF with smaller $m$.

\section{Simulation results}

Let us assume that two thousand nodes are randomly distributed over the circular IEEE 802.11n wireless LAN service area with radius of $r$, and $50 \%$ of nodes are active and attempt to transmit their data frames with payloads of 20,000 bits to an AP, which is located at the center of the service area. It is assumed that the original DCF, the MAC protocol in [6], or the hybrid MAC protocol proposed in the previous section of this paper is employed for the uplink transmission from the nodes to the AP during CPs, and the PCF is employed for the uplink transmissions during CFPs. The transmission and carrier sensing ranges between nodes are assumed to be $r$ and $1.2 r$, respectively. RTS (request to send) and CTS (clear to send) frames are not transmitted, and the data transmissions are unsuccessful only when the collisions between transmissions occur due to the hidden node problem or the concurrent DCF backoff timeouts of the active nodes. Considering low-power WiFi nodes and interference from various traffic sources in IoT traffic environment, we assume the PHY data transfer rate is $6.5 \mathrm{Mbps}$ [7]. Furthermore, the ACK transfer rate is assumed to be $6 \mathrm{Mbps}$, which is a rate of the basic rate set. The values of the parameters used for simulations is presented in Table 1.

The DCF, the MAC protocol in [6] and the hybrid $\mathrm{MAC}$ protocol proposed in this paper were simulated for 
various values of the number $m=20,25,40,50,100$, 200, 250 and 500 of clusters. We generated at least ten thousands packets for each active node and had the packets stored in the transmission buffer of each active node at the start of each simulation, and simulations were conducted until all the packets were successfully transmitted to the AP. In Figure 1, we compare the uplink total MAC throughputs during CPs for the DCF, the MAC protocol in [6] and the hybrid MAC protocol proposed in this paper. Note that the performance of the DCF does not change for various values of $m$.

As can be seen in Fig. 1, compared to the DCF and the MAC protocol in [6], the hybrid MAC protocol proposed in this paper greatly improves the MAC performance. As the number $m$ of clusters becomes greater, the overhead of transmissions of announcement frames becomes larger, which leads to the reduced MAC throughputs of the MAC protocol in [6] and the hybrid MAC protocol proposed in this paper at the large numbers of clusters. As the number of clusters becomes smaller, the MAC throughputs of both the MAC protocol in [6] and the hybrid MAC protocol proposed in this paper follow the roughly bell-shaped curves. When the number of clusters is smaller than 100, the MAC throughputs of both protocols in [6] and proposed in this paper tend to decrease as the number of clusters becomes smaller because the larger number of nodes belonging to each cluster participate in the DCF contention during each SP. However, compared to the hybrid MAC protocol proposed in this paper, the MAC throughput of the MAC protocol in [6] decreases more sharply due to the hidden node problem as the number of clusters becomes smaller.

\section{Conclusions and future research}

We proposed the hybrid MAC protocol combining the basic MAC protocols of IEEE 802.11 wireless LANs to resolve the hidden node problem and improve the MAC performance of IEEE 802.11 wireless LANs. Numerical results were also presented to show that the proposed hybrid MAC protocol greatly improves the MAC through- put. The efficient algorithm for optimizing the number of clusters so that the MAC performance of the hybrid MAC protocol is maximized should be developed by future research.

\section{Acknowledgements}

This paper was supported by research funds from Dong-A university.

\section{REFERENCES}

[1] W.-Y. Choi, "Enhancing MAC Performance of DCF Protocol for IEEE 802.11 Wireless LANs", Journal of Electrical Engineering vol. 68 , no. 1 , 2017, pp. 83-86.

[2] IEEE Wireless LAN Edition, IEEE Press, New York, USA, 2003.

[3] F. Tobagi and L. Kleinrock, "Packet Switching Radio Channels: Part 2 - The Hidden Node Problem Carrier Sense Multiple Access Modes and the Busy Tone Solution", IEEE Transactions on Communications, vol. 23, no. 12, 1975, pp. 1417-1433.

[4] W.-Y. Choi, "Clustering Algorithm for Hidden Node Problem Infrastructure Mode IEEE 802.11 Wireless LANs", in: Proc. of IEEE ICACT 2008.

[5] W. Damayanti, S. Kim and J.-H. Yun, "Collision Chain Mitigation and Hidden Device-Aware Grouping Large-Scale IEEE 802.11ah Networks", Computer Networks, vol. 108, 2016, pp. 296-306.

[6] W.-Y. Choi, "Hybrid MAC Protocol for Improving Performance of IEEE 802.11 Wireless LANs", Journal of Korean Institute of Industrial Engineers vol. 41, no.2, 2015, pp. 220-224 (in Korean).

[7] T. J. Kenney and E. Perahia, "Wireless Device and Method for Low Power and Low Data Rate Operation", United States Patent US 9712362, 2017.

Received 26 June 2018

Woo-Yong Choi was born in Busan, Korea 1970. He received the BS, MS and $\mathrm{PhD}$ degrees in industrial engineering from POSTECH in 1992, 1994 and 1997, respectively. From 1997 to 2001 he was a senior member of technical staff at Hyundai Electronics Industries Co., Ltd.. From 2001 to 2005 he was a senior member of technical staff at ETRI. Since 2005 he has been with Department of Industrial \& Management Systems Engineering at Dong-A University, where he is currently a full tenured professor. 